Ann. Génét. Sél. Anim., I979, 11 (1), 7-I4.

\title{
Détection des gènes responsables de la couleur du plumage chez les volailles espagnoles
}

\author{
J. L. CAMPO et F. OROZCO \\ Departamento de Genética Cuantitativa y Mejora Animal \\ Instituto Nacional de Investigaciones A gravias \\ Carretera de La Coruña, km 7, Madrid (España)
}

\section{Résumé}

Dans le cadre d'un programme de conservation des races de volailles espagnoles en danger d'extinction, mené à la Station Expérimentale de "El Encín " (Madrid), appartenant au Département de Génétique animale de l'I.N.I.A., a été faite l'étude génétique de quelques types de volailles qui n'ont jamais été sélectionnées en race pure, mais qui présentent certaines particularités que l'on essaie de définir et de fixer.

Chez les volailles typiques des Provinces Basques, avec lesquelles le programme a débuté en 1975 , plusieurs types sont définis, le plumage de base étant toujours de couleur dorée. L'un d'eux est caractérisé par la présence de plumes blanches dans la queue, les ailes et le cou; les données obtenues concordent bien avec la supposition que les volailles sont hétérozygotes pour le gène $I$. Un autre type est défini par des phénotypes dorés barrés rarement décrits dans les races de volailles, chez qui le noir barré est pourtant courant; les données suggèrent la présence du gène $B$ lié au sexe.

\section{Introduction}

La nécessité de conserver les ressources génétiques animales ou végétales est, actuellement, largement reconnue; comme le signale la F.A.O. (1975), trois types de motifs justifient cette conservation : pratiques, scientifiques et culturels.

Dans tout programme de conservation, une partie implicite est l'étude génétique des populations concernées, étude qui doit comprendre aussi la définition des gènes qualitatifs les plus caractéristiques de celles-ci. De plus, ceci facilite la participation aux Registres Internationaux de Stocks Génétiques, le principal en aviculture étant celui de Somes (I972).

L'étude des facteurs génétiques qualitatifs est particulièrement intéressante dans les populations multisegrégantes, ce qui se produit toujours quand le patrimoine génétique d'un pays n'est pas encore réparti en races bien définies. C'est le cas des populations de volailles se trouvant en Espagne au Pays Basque; dans ce travail, nous faisons une étude préliminaire d'une partie d'entre elles. 


\section{Matériel et méthodes}

Les volailles utilisées proviennent de divers hameaux des Provinces Basques et appartiennent à un programme de conservation des ressources avicoles espagnoles. Ce programme a débuté en 1975 à la Station Expérimentale de "Eil Encín" (Alcalá de Henares, Madrid) appartenant au Département de Génétique animale de l'Instituto Nacional de Investigaciones Agrarias (I.N.I.A.). Bien que ces volailles n'aient jamais été sélectionnées pour former une race pure, elles constituent des types particuliers de la région.

La couleur de base du plumage est doré avec des intensités variées, avec des plumes noires bien définies particulièrement sur la queue, les rémiges de l'aile et le cou (fig. IA).
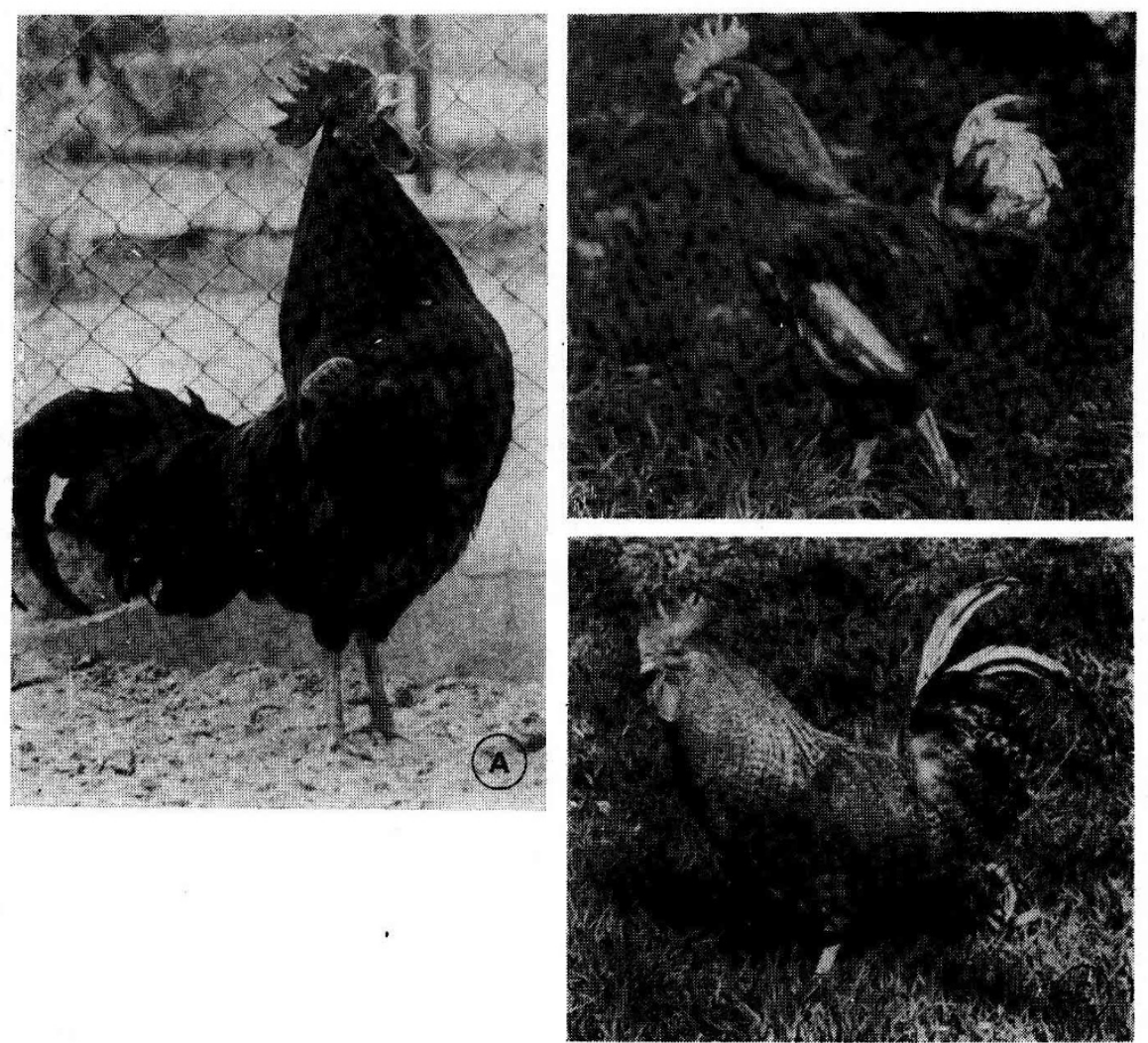

Fig. x. - Variants colorés dans le Pays Basque espagnol.

A) Coq doré avec des plumes noires sur la queue, l'aile et le cou.

B) Coq doré avec des plumes blanches sur la queue, l'aile et le cou.

C) Coq barré et doré.

Fowel colour variants in Spanish Basque Country.

A) Golden cockerel with black feathers on tail, wing and hackle.

B) Golden cockerel with white feathers on tail, wing and hackle.

C Barred golden cockerel. 
Il existe de nombreuses variantes de ce plumage de base, mais nous n'allons ici en considérer que deux. Chez l'une d'elles il apparaît des zones blanches dans le plumage doré, avec cette particularité que dans les cas les plus marqués 1'inhibition de la couleur n'apparaît que dans les zones noires du plumage de base (fig. IB)

L'autre variante est un plumage doré barré, difficile à apprécier parfois chez. les femelles, bien qu'elles le présentent généralement sur la poitrine et le cou. Les mâles barrés typiques sont d'un doré intense et nettement barré; ils conservent le pigment noir du type de base, particulièrement sur la queue et les ailes où les plumes sont barrées de noir et de blanc (fig. IC).

Bien que le nombre de volailles de départ soit légèrement supérieur, la génération parentale, pour cette étude, a été formée de 5 mâles et 25 femelles dorés avec des plumes blanches et de 4 mâles et 20 femelles à plumage doré barré.

Nous avons fait trois accouplements consécutifs, dans des parcs de un mâle et plusieurs femelles, avec deux lots dans chacun, séparés de ro jours. Les naissances eurent lieu entre septembre et décembre I977, donnant 255 volailles aptes à l'analyse dans le cas des plumes blanches et I72 dans celui des plumes barrées.

I a comparaison entre les proportions observées et attendues a été faite avec un test de $\chi^{2}$, en imposant la condition que les fréquences absolues attendues devaient être supérieures à 5. Pour les cas compris entre 5 et ro, avec un degré de liberté, il n'a pas été nécessaire de faire la correction pour la continuité étant donné que les valeurs de $\chi^{2}$ furent très faibles. Bien que 1'on ait fait les $\chi^{2}$ partiels afin d'obtenir une information plus large, nous incluons trois types de $\chi^{2}$ globaux : somme des $\chi^{2}$ partiels, "pool " des données et hétérogénéité (SNEDECOR, I956).

Pour tout ce qui concerne la description et la nomenclature des gènes, nous avons suivi HuTT (I949), actualisé par Somes (I972).

\section{Résultats et discussion}

\section{Phénotypes avec des plumes blanches}

Les ségrégations phénotypiques observées quand on accouple des mâles dorés à plumes blanches avec des femelles de même phénotype sont rapportées dans le tableau I, ainsi que le nombre de femelles accouplées à chaque mâle. Étant donné que dans les descendances il apparaît toujours des animaux dorés sans plumes blanches, les mâles et les femelles parentaux doivent être hétérozygotes pour un gène inhibiteur de la couleur.

L'ajustement à la ségrégation théorique : 3 dorés à plumes blanches et $\mathrm{I}$ doré normal est satisfaisant pour tous les résultats partiels, ainsi que pour le " pool " des données et pour le $\chi^{2}$ somme des partiels.

Il n'est pas difficile, à l'intérieur des phénotypes à plumes blanches, de distinguer deux groupes; l'un est semblable aux parents utilisés pour les accouplements; chez l'autre, le plumage blanc est très étendu sur tout le corps, jusqu'à dominer sur les zones de plumage doré, qui peuvent ainsi se trouver réduites à quelques taches.

Ceci conduit à l'hypothèse que le gène inhibiteur de la couleur a un effet plus drastique chez les homozygotes que chez les hétérozygotes. D'autre part, étant donné que le type de départ a des plumes noires sur la queue et les ailes, certainement dues à la présence du gène pour le plumage "columbian " $\left(C_{0}\right)$ et qu'ici il en apparaît des blanches, on peut conclure que l'inhibition est plus marquée sur les plumes noires que sur les dorées. 
TABIEAU I

Ségrégations phénotypiques observées dans les accouplements de mâles et de femelles à plumes blanches

Phenotypic segregations in crossing males and females with white feathers

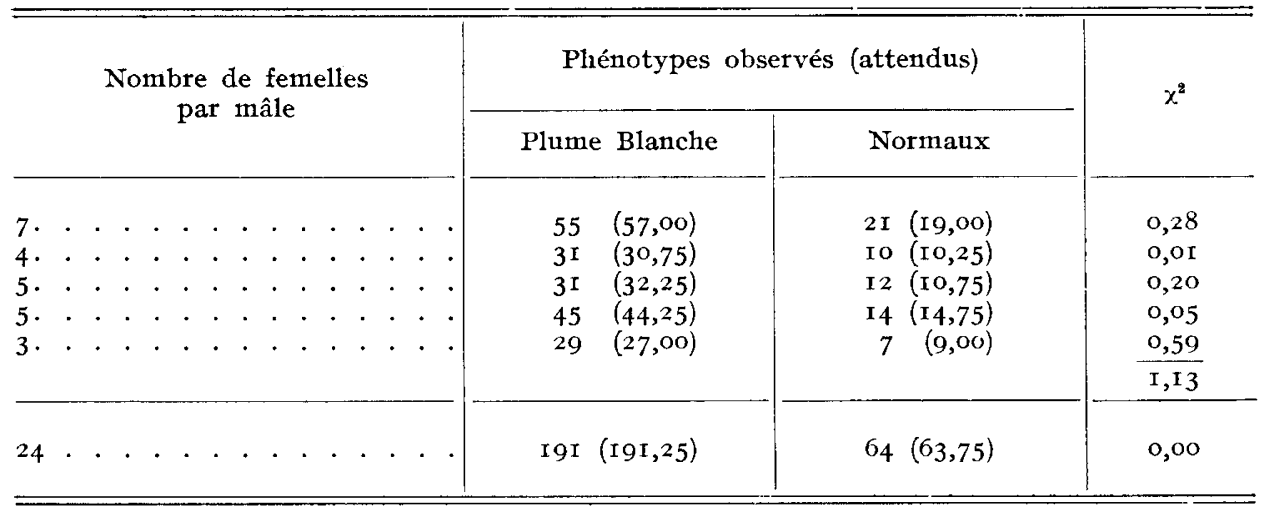

Tout ceci concorde avec le type d'action du gène $I$ (blanc dominant), présent chez plusieurs variétés de nombreuses races, dont la Leghorn Blanche.

Dans le tableau 2 nous avons reporté les proportions phénotypiques du tableau I en considérant les deux groupes parmi ceux à plumage à zones blanches, selon qu'elles apparaissent seulement sur la queue et l'extrémité des ailes (Ii), ou prédominent nettement sur les zones dorées $(I I)$.

\section{TABLEAU 2}

Ségrégations génotypiques correspondant aux phénotypes du tableau I Genotypic segregations corresponding to phenotypes of table I

\begin{tabular}{|c|c|c|c|}
\hline \multicolumn{3}{|c|}{ Génotypes observés (attendus) } & \multirow{2}{*}{$\chi^{2}$} \\
\hline$I I$ & $I i$ & $i i$ & \\
\hline $\begin{array}{rr}\text { I4 } & (19,00) \\
\text { I3 } & (10,25) \\
\text { Io } & (10,75) \\
22 & (14,75) \\
\text { Io } & (9,00)\end{array}$ & $\begin{array}{ll}4 \mathrm{I} & \left(3^{8}, 00\right) \\
\text { I8 } & \left(20,5^{\circ}\right) \\
\text { 2 I } & \left(2 \mathrm{I}, 5^{\circ}\right) \\
23 & \left(29,5^{\circ}\right) \\
\text { I9 } & (18,00)\end{array}$ & $\begin{array}{rr}\text { 2I } & (\mathrm{r} 9,00) \\
\text { Io } & (\mathrm{ro}, 25) \\
\text { I2 } & (\mathrm{I} 0,75) \\
\text { I4 } & (\mathrm{I} 4,75) \\
7 & (9,00)\end{array}$ & $\begin{array}{l}\mathrm{I}, 77 \\
\mathrm{I}, 05 \\
0,2 \mathrm{I} \\
\left.5,03 \quad \quad^{*}\right) \\
0,6 \mathrm{I}\end{array}$ \\
\hline $69(63,75)$ & I $22\left(\right.$ I $\left.27,5^{\circ}\right)$ & $64(63,75)$ & 0,67 \\
\hline
\end{tabular}

(*) Significatif à ro p. roo.

$I I=$ Homozygotes à plume blanche (Homozygote white feather).

$I i=$ Hétérozygotes à plume blanche (Heterozygote white feather).

$i i=$ Normaux (Normal). 
L'ajustement à la ségrégation théorique $I I, 2 I i$, rii est correct pour le "pool" de toutes les données et pour la somme des $\chi^{2}$ partiels, bien que le $\chi^{2}$, pour l'hétérogénéité soit significatif au niveau $5 \mathrm{p}$. Ioo $\left(\chi^{2}{ }_{3}=8,00\right)$. Seul le $\chi^{2}$ pour l'une des ségrégations partielles est significatif, au niveau Io p. I0O, mais si on fait 1'ajustement à l'intérieur du groupe à plumes blanches $(\mathrm{I} I I, 2 I i)$ il devient significatif au niveau 5 p. Ioo $\left(\chi^{2}{ }_{1}=4,99\right)$, aucun des $\chi^{2}$ globaux n'étant significatif.

Ainsi, il paraît évident que la seule différence entre le phénotype à plumes blanches décrit dans cette population et le type doré normal réside dans la présence du gène $I$. $\mathrm{D}$ ₹ même, si le type normal est courant et apparaît dans de nombreuses races (Brahma Fauve, Cornish Rouge, New Hampshire, Rhode Island Rouge, Sussex Rouge, Wyandotte Columbian Fauve), celui à plumes blanches n'est signalé que dans la race Brahma (variété Bright).

\section{Phénotypes barrés}

En ce qui concerne les volailles à plumage doré barré, les ségrégations phénotypiques observées quand on accouple des mâles et des femelles de ce phénotype sont rapportées dans le tableau 3.

TABIEAU 3

Ségrégations phénotypiques observées dans les accouplements entre mâles et femelles barrés Phenotypic segregations in crossing barred males and temales

\begin{tabular}{|c|c|c|c|c|c|c|c|}
\hline & \multirow{3}{*}{\multicolumn{2}{|c|}{$\begin{array}{l}\text { Nombre } \\
\text { de femelles } \\
\text { par mâle }\end{array}$}} & \multicolumn{4}{|c|}{ Phénotypes observés (attendus) } & \multirow{3}{*}{$x^{2}$} \\
\hline & & & \multicolumn{2}{|c|}{ Mâles barrés } & \multicolumn{2}{|c|}{ Femelles } & \\
\hline & & & Sombres & Clairs & Barrées & Normales & \\
\hline 5 &. & . & $7(12,00)$ & $14(12,00)$ & IO $(12,00)$ & I 7 (I 2,Oo) & 4,82 \\
\hline 9 & . . . . & . . & I6 (I 6,75$)$ & I $6($ I 6,75$)$ & I $2(\mathrm{I} 6,75)$ & $23(\mathrm{r} 6,75)$ & 3,74 \\
\hline 3 & . . . . . & . . & $5(6,25)$ & $6(6,25)$ & $5(6,25)$ & $9(6,25)$ & 1,72 \\
\hline 3 & . . . . . . & . . & $8(8,00)$ & $7(8,00)$ & Io $(8,00)$ & $7(8,00)$ & 0,76 \\
\hline & & & & & & & $\overline{\mathrm{II}, 04}(*)$ \\
\hline 20 & . . . . & . . & $36(43,00)$ & $43(43,00)$ & $37(43,00)$ & $5^{6}(43,00)$ & $5,9 \mathrm{I}$ \\
\hline
\end{tabular}

$\left({ }^{*}\right)$ Significatif à 5 p. roo.

Cinez les femelles, on obtient des phénotypes barrés et non barrés. Tous les mâles obtenus étaient barrés, avec cette particularité qu'on pouvait les classer en deux groupes : "sombres" comme leurs parents avec une coloration dorée intense et "clairs " avec une coloration très pâle et faible, et même avec une proportion de blanc plus importante dans le barré. Ceci suggère clairement que la forme sombre est hétérozygote et la forme claire homozygote pour le caractère barré. 
L'ajustement à la ségrégation théorique : I mâle sombre, I mâle clair, I femelle barrée, I femelle normale, est correct pour le " pool " des données bien que le $\chi^{2}$ somme des partiels soit significatif au niveau $5 \mathrm{p}$. Ioo. Le $\chi^{2}$ pour l'hétérogénéité $\left(\chi^{2}{ }_{1}=5, \mathrm{r} 3\right)$ est aussi significatif à 5 p. IoO.

Pour approfondir un peu l'analyse, nous avons vérifié l'ajustement des femelles et des mâles séparément (tableau 4). Dans tous les cas, la proportion de mâles barrés clairs et sombres était celle, attendue, de $I: I$. Chez les femelles ( $I: I$ aussi) un des $\chi^{2}$ partiels est significatif au niveau ro p. IOO $\left(\chi^{2}{ }_{1}=3,46\right)$; le $\chi^{2} d u$ "pool" l'est au niveau 5 p. $100\left(\chi_{1}^{2}=3,88\right)$, indiquant un excès de femelles non barrées, ce qui peut être dû̀ à la difficulté de distinguer dans quelques cas le phénotype barré chez les femelles. Ni le $\chi^{2}$ total $(6,95$ avec 4 degrés de liberté), ni celui pour l'hétérogénéité $(3,07$ avec 3 degrés de liberté) ne sont significatifs.

\section{TABLEAU 4}

Ajustement indépendant pour les mâles et pour les femelles des proportions obtenues au tableau 3 Independant adjustments for males and females of ratios from table 3

\begin{tabular}{|c|c|c|c|c|c|}
\hline \multicolumn{2}{|c|}{ Mâles } & \multirow{2}{*}{$\chi^{2}$} & \multicolumn{2}{|c|}{ Femelles } & \multirow{2}{*}{$\chi^{2}$} \\
\hline Sombres & Clairs & & Barrées & Normales & \\
\hline $7($ (o,5) & I $4(10,5)$ & 2,34 & Io $(\mathrm{I} 3,5)$ & I $7($ I 3,5$)$ & $\mathrm{I}, 82$ \\
\hline $16(16,0)$ & I6 (I 6,0$)$ & 0,00 & I $2(\mathrm{I} 7,5)$ & $23(17,5)$ & $3,46(* *)$ \\
\hline $5(5,5)$ & $6(5,5)$ & 0,10 & $5(7,0)$ & $9(7,0)$ & $\mathrm{I} \mathrm{r}_{4}$ \\
\hline \multirow[t]{2}{*}{$8(7,5)$} & $7(7,5)$ & 0,06 & Io $(8,5)$ & $7(8,5)$ & 0,53 \\
\hline & & $\overline{2,50}$ & & & $\overline{6,95}$ \\
\hline $36(39,5)$ & $43(39,5)$ & 0,62 & $37\left(4^{6}, 5\right)$ & $5^{6}\left(4^{6}, 5\right)$ & $3,88 \quad(*)$ \\
\hline
\end{tabular}

${ }^{*}$ ) Significatif à 5 p. Ioo.

(**) Significatif à ro p. roo.

Tout ceci concorde avec la forme d'action du gène $B$ (barré lié au sexe), typiquement décrit dans la race Plymouth Rock Barrée et présent dans de nombreuses races.

Pour confirmer ce point de vue, nous avons fait, avec un seul accouplement en deux lots, des croisements entre mâles barrés sombres $(B b)$ et des femelles normales $(b-)$, et réciproquement entre des mâles normaux $(b b)$ et des femelles barrées $(B-)$. Les ségrégations obtenues sont présentées dans le tableau 5; en raison du faible effectif pour des ségrégations partielles, nous avons seulement calculé le $\gamma^{2}$ pour le " pool " des données. L'ajustement est satisfaisant dans les deux cas (I : I : I : I dans les premiers cas et seulement des mâles barrés et des femelles normales dans le second).

I1 faut indiquer que dans les Catalogues de Races Internationaux, aucune race à plumage barré doré n'est décrite, bien que celles barrées de noir et, dans une moindre mesure, celles barrées de doré et noir soient fréquentes. Seuls PunNeTt et 
TABLEAU 5

Ségrégations phénotypiques observées dans les accouplements entre mâles barrés et femelles normales ( $\mathrm{b}-$ ), ainsi que dans les accouplements réciproques

Phenotypic segregations in crossing barred males (Bb) and normal females (b-) and reciprocal crossings

\begin{tabular}{|c|c|c|c|c|c|c|}
\hline \multirow{3}{*}{$\begin{array}{c}\text { Type } \\
\text { d'accou- } \\
\text { plement }\end{array}$} & \multirow{3}{*}{$\begin{array}{l}\text { Nombre de } \\
\text { femelles } \\
\text { par mâle }\end{array}$} & \multicolumn{4}{|c|}{ Phénotypes observés (attendus) } & \multirow{3}{*}{$x^{2}$} \\
\hline & & \multicolumn{2}{|c|}{ Mâles } & \multicolumn{2}{|c|}{ Femelles } & \\
\hline & & Barrés & Normaux & Barrées & Normales & \\
\hline $\begin{array}{l}B b \times b-. \\
b b \times B-.\end{array}$ & $\begin{array}{l}6 \\
8\end{array}$ & $\begin{array}{ll}\text { I9 } & (13,25) \\
\text { 3I } & (35,50)\end{array}$ & ro $(13,25)$ & II $(\mathrm{I} 3,25)$ & $\begin{array}{ll}\text { I3 } & (13,25) \\
40 & (35,50)\end{array}$ & $\begin{array}{l}3,68 \\
\mathrm{I}, \mathrm{I}_{4}\end{array}$ \\
\hline
\end{tabular}

PEASE ont décrit des phénotypes barrés dorés, appartenant à la variété Gold Barred Plymouth Rock (I928) et à la Chamoise Campine (I92I); le premier était dû au facteur barré lié au sexe $(B)$, et le second au barré autosomal $(A b)$. Nous ne pouvons pas encore dire si le gène présent dans la population étudiée ici est celui typique de la Plymouth Rock Barrée ou si, comme le considéraient Punnetr et PEASE (r928), c'est un de ses allèles, bien que ce ne pourrait être que l'effet du gène qui serait distinct chez les volailles dorées.

Rę̧u pour publication en mai 1979.

\section{Remerciements}

Nous remercions G. BoLET (Station de Génétique Quantitative, Jouy-en-Josas) pour la mise au point de la traduction française du manuscrit.

\section{Summary}

\section{Detection of the plumage color genes in Spanish chickens}

In a conservation program of Spanish chickens breeds carried out at Experimental Station of "El Encin" (Madrid, Spain), is included the genetical study of some domestic fowl types that, though they have not been as yet selected as a breed, have some distinctive traits that we are trying to define and to fix.

The program was started in 1975 with birds from the Basque country, where there are several types all with golden color as basic plumage pattern. One of them is characterized by the presence of white feathers on the tail, wings and hackle; the obtained results agree with the assumption that the birds have in heterozygous state the $I$ gene (dominant white). Another type is defined by golden barred phenotypes that have rarely been described in chicken breeds, where the black barred phenotype is however very usual. The results suggest the presence of the sex-linked barring gene $(B)$. 


\section{Références bibliographiques}

I*A.O., I975. Étude pilote sur la conservation des ressources génétiques animales. Programme des Nations Unies pour l'Environnement, Organisation des Nations Unies pour l'Alimentation et l'Agriculture (F.A.O.), Rome, 70 p.

HUTT F. B., 1949. Genetics of the Fowl. McGraw-Hill, New-York. I-590.

Punneti R. C., Pease M. S., r92I. Genetic studies in poultry. IV. On the barred plumage of certain breeds. J. Genetics, 11, 235-240.

Punnet't R. C., Pease M. S., 1928. Genetic studies in poultry. VI. The Gold Barred Rock. $J$. Genetics, 19, 337-350.

SNedecor G. W., I956. Statistical Methods. The Iowa State College Press, Ames, 2 I 2 -2 I9.

SOMES R. G., I972. Registry of poultry genetic stocks in North America. Storrs Agricultural Experiment Station, The University of Connecticut, Storrs. 